\title{
Erratum to: Efficacy and Safety of IgPro20, a Subcutaneous Immunoglobulin, in Japanese Patients with Primary Immunodeficiency Diseases
}

\author{
Hirokazu Kanegane • Kohsuke Imai • Masafumi Yamada • Hidetoshi Takada • \\ Tadashi Ariga - Martin Bexon • Mikhail Rojavin • Wilson Hu • Midori Kobayashi • \\ John-Philip Lawo • Shigeaki Nonoyama • Toshiro Hara • Toshio Miyawaki
}

Published online: 2 April 2014

(C) Springer Science+Business Media New York 2014

\section{Erratum to: J Clin Immunol (2013) 6:169-178 \\ DOI 10.1007/s10875-013-9985-z}

The corresponding author of this article has been changed to Hirokazu Kanegane, University of Toyama, Department of Pediatrics, Graduate School of Medicine and Pharmaceutical Sciences, Toyama, Japan. Email: kanegane@med.u-toyama.ac.jp.

The online version of the original article can be found at http://dx.doi..org/ 10.1007/s10875-013-9985-z.

H. Kanegane $(\bowtie)$

Department of Pediatrics, Graduate School of Medicine and Pharmaceutical Sciences, University of Toyama, Toyama, Japan

e-mail: kanegane@med.u-toyama.ac.jp

K. Imai

Department of Community Pediatrics, Perinatal and Maternal

Medicine, Tokyo Medical and Dental University, Tokyo, Japan

M. Yamada $\cdot$ T. Ariga

Department of Pediatrics, Hokkaido University Graduate, School of

Medicine, Sapporo, Japan

H. Takada $\cdot$ T. Hara

Department of Pediatrics, Graduate School of Medical Sciences,

Kyushu University, Fukuoka, Japan

M. Bexon

CSL Behring AG, Berne, Switzerland

M. Rojavin

CSL Behring LLC, King of Prussia, PA, USA
W. Hu

CSL Limited, Research and Development, Melbourne, Victoria, Australia

M. Kobayashi

CSL Behring KK, Tokyo, Japan

J.-P. Lawo

CSL Behring GmbH, Marburg, Germany

S. Nonoyama

Department of Pediatrics, National Defense Medical College,

Saitama, Japan

\section{T. Miyawaki}

Toyama City Hospital, Toyama, Japan

e-mail: toshio65@tch.toyama.toyama.jp 